\title{
Performance Analysis of the Home M2M Cooperative Networks
}

\author{
Lingwei Xu ${ }^{1}$, Hao Zhang ${ }^{1,2}$, Tingting $\mathrm{Lu}^{1}$ and $\mathrm{T}$ A Gulliver ${ }^{2}$ \\ ${ }^{1}$ College of Information Science and Engineering, Ocean University of China, \\ Qingdao 266100, China \\ ${ }^{2}$ Department of Electrical and Computer Engineering, University of Victoria, \\ Victoria V8W 3P6, Canada \\ gaomilaojia2009@163.com; zhanghao@ouc.edu.cn; lvtingting33@163.com \\ agullive@ece.uvic.ca
}

\begin{abstract}
Based on decode-and-forward (DF) relaying scheme, the exact closed-form expression of the outage probability (OP) for the home mobile-to-mobile (M2M) cooperative networks over $N$-Nakagami fading channels is derived in this paper. Then the $O P$ performance under different conditions is evaluated through numerical simulations. The numerical simulation results coincide with the theoretical results well, and the accuracy of the theoretical results is verified. The simulation results showed that: the fading coefficient, the number of cascaded components, the relative geometrical gain, and the power-allocation parameter have an important influence on the OP performance.
\end{abstract}

Keywords: home M2M communication, N-Nakagami fading channels, decode-andforward, outage probability

\section{Introduction}

Mobile-to-mobile (M2M) communication has attracted wide research interest in recent years. It is widely employed in many popular wireless communication systems, such as mobile ad-hoc networks and home networks [1]. Home M2M networks have been steadily gaining popularity especially in homes and office spaces. It allows multiple users to exchange information, share data, interact with ideas, and cooperate on common goals across geographical and time boundaries [2]. The higher simplicity and affordability of home M2M networks are achieved through the connectivity of devices, such as smartphones, PDAs and tablets [3]. When the devices are in motion, the double-Rayleigh fading model has been shown to be applicable [4]. Extending this model to the more realistic Nakagami fading, a double-Nakagami fading model has also been considered [5]. The moments-generating, probability density, cumulative distribution, and moments functions of the N-Nakagami distribution are developed in closed form using the Meijer's G-function [6].

Cooperative diversity has been proposed as a promising solution for the high data-rate coverage required in home M2M networks. In [7], the author derived an exact and closedform expression for outage probability (OP) of opportunistic amplify-and-forward (AF) relaying over Nakagami-m fading channels. Similar research is done just based on selection decode-and-forward (DF) relaying scheme in [8]. Based on AF relaying scheme, [9] investigated pairwise error probability (PEP) for the cooperative inter-vehicular communication (IVC) system over double-Nakagami fading channels. Based on DF relaying scheme, [10] investigated the exact symbol error rate (SER) and asymptotic SER expressions of the M2M system by using the widely studied moment generating function (MGF) approach over double-Nakagami fading channels.

However, to the best knowledge of the author, no previous work has been reported in the literature on OP performance of the home M2M cooperative networks over $\mathrm{N}$ - 
Nakagami fading channels. In the present work, we extend our analysis for N-Nakagami case which subsumes double-Nakagami in $[9,10]$ as special cases. Based on DF relaying scheme, the exact closed-form OP expression for the home M2M cooperative networks over N-Nakagami fading channels is derived.

The rest of the paper is organized as follows. The home M2M cooperative networks model is presented in Section 2. Section 3 provides the exact closed-form OP expression for the home M2M cooperative networks. Section 4 conducts Monte Carlo simulations to verify the analytical results. Concluding remarks are given in Section 5.

\section{The System Model}

We consider a three-node cooperation model, namely mobile source (MS), mobile relay (MR), and mobile destination (MD) nodes. The nodes operate in half-duplex mode, which are equipped with a single pair of transmitter and receiver antennas.

According to [9], we let $d_{\mathrm{SD}}, d_{\mathrm{SR}}$, and $d_{\mathrm{RD}}$ represent the distances of source-todestination $(\mathrm{MS} \rightarrow \mathrm{MD})$, source-to-relay $(\mathrm{MS} \rightarrow \mathrm{MR})$, and relay-to-destination $(\mathrm{MR} \rightarrow \mathrm{MD})$ links, respectively. Assuming the path loss between MS $\rightarrow$ MD to be unity, the relative gain of $\mathrm{MS} \rightarrow \mathrm{MR}$ and $\mathrm{MR} \rightarrow \mathrm{MD}$ links are defined as $G_{\mathrm{SR}}=\left(d_{\mathrm{SD}} / d_{\mathrm{SR}}\right)^{v}$ and $G_{\mathrm{RD}}=$ $\left(d_{\mathrm{SD}} / d_{\mathrm{RD}}\right)^{v}$, respectively, where $v$ is the path loss coefficient[11]. We further define the relative geometrical gain $\mu=G_{\mathrm{SR}} / G_{\mathrm{RD}}$ (in decibels), which indicates the location of the relay with respect to the source and destination. When the relay is close to the destination node, the values of $\mu$ are negative. When the relay is close to the source node, the values of $\mu$ are positive. When the relay has the same distance to the source and destination nodes, $\mu$ is $0 \mathrm{~dB}$.

Let $h_{\mathrm{SD}}, h_{\mathrm{SR}}$, and $h_{\mathrm{RD}}$ represent the complex channel coefficients of $\mathrm{MS} \rightarrow \mathrm{MD}$, MS $\rightarrow$ $\mathrm{MR}$, and MR $\rightarrow$ MD links, respectively, which follow N-Nakagami distribution. $h_{\mathrm{SD}}, h_{\mathrm{SR}}$, and $h_{\mathrm{RD}}$ are assumed to be a product of statistically independent, but not necessarily identically distributed, $N$ independent random variables

$$
h=\prod_{i=1}^{N} a_{i}
$$

where $N$ is the number of cascaded components. $a_{l}$ is a Nakagami distributed random variable with probability density function (PDF)

$$
f(a)=\frac{2 m^{m}}{\Omega^{m} \Gamma(m)} a^{2 m-1} \exp \left(-\frac{m}{\Omega} a^{2}\right)
$$

where $\Gamma(\cdot)$ is the Gamma function, $m$ is the fading coefficient and $\Omega$ is a scaling factor.

The PDF of $h$ is given by[4]

$$
f_{h}(h)=\frac{2}{h \prod_{i=1}^{N} \Gamma\left(m_{i}\right)} G_{0, N}^{N, 0}\left[\left.h^{2} \prod_{i=1}^{N} \frac{m_{i}}{\Omega_{i}}\right|_{m_{1}, \ldots, m_{N}} ^{-}\right]
$$

where $\mathrm{G}[\cdot]$ is the Meijer's $G$-function.

Let $y_{\mathrm{SD}}=\left|h_{\mathrm{SD}}\right|^{2}, \quad y_{\mathrm{SR}}=\left|h_{\mathrm{SR}}\right|^{2}$, and $y_{\mathrm{RD}}=\left|h_{\mathrm{RD}}\right|^{2}$. The corresponding cumulative density functions (CDF) of $y$ can be derived as[4]

$$
F_{y}(y)=\frac{1}{\prod_{i=1}^{N} \Gamma\left(m_{i}\right)} G_{1, N+1}^{N, 1}\left[\left.y \prod_{i=1}^{N} \frac{m_{i}}{\Omega_{i}}\right|_{m_{1}, \ldots, m_{N}, 0} ^{1}\right]
$$

By taking the first derivative of (4) with respect to $y$, the corresponding PDF can be obtained as [4] 


$$
f_{y}(y)=\frac{1}{y \prod_{i=1}^{N} \Gamma\left(m_{i}\right)} G_{0, N}^{N, 0}\left[\left.y \prod_{i=1}^{N} \frac{m_{i}}{\Omega_{i}}\right|_{m_{1}, \ldots, m_{N}} ^{-}\right]
$$

Based on the DF cooperation protocol, the received signals $r_{\mathrm{SD}}$ and $r_{\mathrm{SR}}$ at the MR and the MD during the first time slot can be written as [7]

$$
\begin{gathered}
r_{S D}=\sqrt{K E} h_{S D} x+n_{D} \\
r_{S R}=\sqrt{G_{S R} K E} h_{S R} x+n_{S R}
\end{gathered}
$$

where $x$ denotes the transmitted signal, $n_{S \mathrm{R}}$ and $n_{\mathrm{D}}$ are the zero-mean complex Gaussian random variables with variance $N_{0} / 2$ per dimension. Here, $E$ is the total energy which is used by both source and relay terminals during two time slots. $K$ is the power-allocation parameter that controls the fraction of power reserved for the broadcasting phase. If $K=0.5$, the equal power allocation (EPA) scheme is used.

During the second time slot, the relay terminal decodes its received signal and transmits the signal if it is correctly decoded. The received signal at the destination is therefore given by

$$
r_{R D}=k \sqrt{G_{R D}(1-K) E} h_{R D} x+n_{R D}
$$

where $k=1$, the symbol is correctly decoded, otherwise $k=0 . n_{\mathrm{RD}}$ is a conditionally zeromean complex Gaussian random variable with variance $N_{0} / 2$ per dimension.

If selection combining (SC) method is used at the MD, the output SNR at the destination can then be calculated as [11]

where

$$
\gamma_{S C}=\max \left(\gamma_{S D}, k \gamma_{R D}\right)
$$

$$
\begin{gathered}
\gamma_{S D}=\frac{K\left|h_{S D}\right|^{2} E}{N_{0}}=K\left|h_{S D}\right|^{2} \bar{\gamma} \\
\gamma_{R D}=\frac{G_{R D}(1-K)\left|h_{R D}\right|^{2} E}{N_{0}}=G_{R D}(1-K)\left|h_{R D}\right|^{2} \bar{\gamma}
\end{gathered}
$$

\section{The OP for the Home M2M Cooperative Networks}

In this section, the closed-form OP expression for the home M2M cooperative networks is derived.

The mutual information between the source and relay is

$$
I_{S R}=\frac{1}{2} \log _{2}\left(1+\gamma_{S R}\right)
$$

where

$$
\gamma_{S R}=\frac{G_{S R} K\left|h_{S R}\right|^{2} E}{N_{0}}=G_{S R} K\left|h_{S R}\right|^{2} \bar{\gamma}
$$

If the mutual information $I_{\mathrm{SR}}$ is lower than spectral efficiency $R$, the relay will not be used for cooperation. It can be denoted as

$$
P_{\text {outsr }}=\operatorname{Pr}\left(I_{S R}<R\right)=\operatorname{Pr}\left(\frac{1}{2} \log _{2}\left(1+\gamma_{S R}\right)<R\right)
$$

So the mutual information of the destination received during two time slots $I_{\mathrm{DF}}$ can be denoted as

$$
I_{D F}=\frac{1}{2} \log _{2}\left(1+\gamma_{S D}\right)
$$

If the mutual information $I_{\mathrm{SR}}$ is not lower than spectral efficiency $R$, the relay will be 
used for cooperation. $I_{\mathrm{DF}}$ is given as

$$
I_{D F}=\frac{1}{2} \log _{2}\left(1+\gamma_{S C}\right)=\frac{1}{2} \log _{2}\left(1+\max \left(\gamma_{S D}, \gamma_{R D}\right)\right)
$$

The outage probability of the home M2M networks can be expressed as

$$
\begin{aligned}
& P_{\text {out }}=\operatorname{Pr}\left(I_{S R}<R\right) \operatorname{Pr}\left(\frac{1}{2} \log _{2}\left(1+\gamma_{S D}\right)<R\right)+ \\
& \operatorname{Pr}\left(I_{S R} \geq R\right) \operatorname{Pr}\left(\frac{1}{2} \log _{2}\left(1+\max \left(\gamma_{S D}, \gamma_{R D}\right)\right)<R\right) \\
& =I_{1}+I_{2}
\end{aligned}
$$

From Appendix A, we present a new closed-form OP expression as

$$
\begin{aligned}
& P_{\text {out }}=\frac{1}{\prod_{j=1}^{N} \Gamma\left(m_{j}\right) \prod_{j j=1}^{N} \Gamma\left(m_{j j}\right)} G_{1, N+1}^{N, 1}\left[\frac{\gamma_{\text {th }}}{\overline{\gamma_{S R}}} \prod_{j=1}^{N} \frac{m_{j}}{\Omega_{j}}||_{m_{1}, \ldots, m_{N}, 0}\right] G_{1, N+1}^{N, 1}\left[\frac{\gamma_{t h}}{\overline{\gamma_{S D}}} \prod_{j j=1}^{N} \frac{m_{j j}}{\Omega_{j j}}||_{m_{1}, \ldots, m_{N}, 0}\right] \\
& +\frac{1}{\prod_{j j=1}^{N} \Gamma\left(m_{j j}\right) \prod_{j j j=1}^{N} \Gamma\left(m_{j j j}\right)}\left(1-\frac{1}{\prod_{j=1}^{N} \Gamma\left(m_{j}\right)} G_{1, N+1}^{N, 1}\left[\left.\frac{\gamma_{t h}}{\frac{\gamma_{S R}}{\prod_{j=1}}} \prod_{j} \frac{m_{j}}{\Omega_{j}}\right|_{m_{1}, \ldots, m_{N}, 0} ^{1}\right] \times\right. \\
& G_{1, N+1}^{N, 1}\left[\left.\frac{\gamma_{t h}}{\frac{\gamma_{S D}}{\gamma_{j j}}} \prod_{j=1}^{N} \frac{m_{j j}}{\Omega_{j j}}\right|_{m_{1}, \ldots, m_{N}, 0} ^{1}\right] G_{1, N+1}^{N, 1}\left[\left.\frac{\gamma_{t h}}{\overline{\gamma_{R D}}} \prod_{j j j=1}^{N} \frac{m_{j j j}}{\Omega_{j j j}}\right|_{m_{1}, \ldots, m_{N}, 0} ^{1}\right]
\end{aligned}
$$

\section{Numerical Results}

In this section, some numerical results are presented to illustrate and verify the OP results obtained in the previous sections.

Figure 1 presents the OP performance of the home M2M cooperative networks over NNakagami fading channels. The relative geometrical gain $\mu=0 \mathrm{~dB}$. The power-allocation parameter $K=0.5$. The given threshold $\gamma_{\mathrm{th}}=-4 \mathrm{~dB}$. Here, we consider the following scenarios based on the combinations of the number of cascaded components $N$ and fading coefficient $m$ :

(1) Scenario 1: $m_{\mathrm{SD}}=1, m_{\mathrm{SR}}=1, m_{\mathrm{RD}}=1$ and $N_{\mathrm{SD}}=N_{\mathrm{SR}}=N_{\mathrm{RD}}=2$.

(2)Scenario 2: $m_{\mathrm{SD}}=2, m_{\mathrm{SR}}=2, m_{\mathrm{RD}}=2$ and $N_{\mathrm{SD}}=N_{\mathrm{SR}}=N_{\mathrm{RD}}=2$.

(3) Scenario 3: $m_{\mathrm{SD}}=3, m_{\mathrm{SR}}=3, m_{\mathrm{RD}}=3$ and $N_{\mathrm{SD}}=N_{\mathrm{SR}}=N_{\mathrm{RD}}=2$.

From Figure 1, we can obtain that the numerical simulation results coincide with the theoretical results well, and the accuracy of the theoretical OP is verified. Simulation results show that the OP performance is improved with the fading coefficient $m$ increased. For example, when SNR $=10 \mathrm{~dB}, m=1$, the $\mathrm{OP}$ is $2 \times 10^{-2}, m=2$, the $\mathrm{OP}$ is $1 \times 10^{-3}$, $m=3$, the OP is $3 \times 10^{-5}$. When the $m$ is fixed, with the increase of SNR, the OP is reduced gradually. 


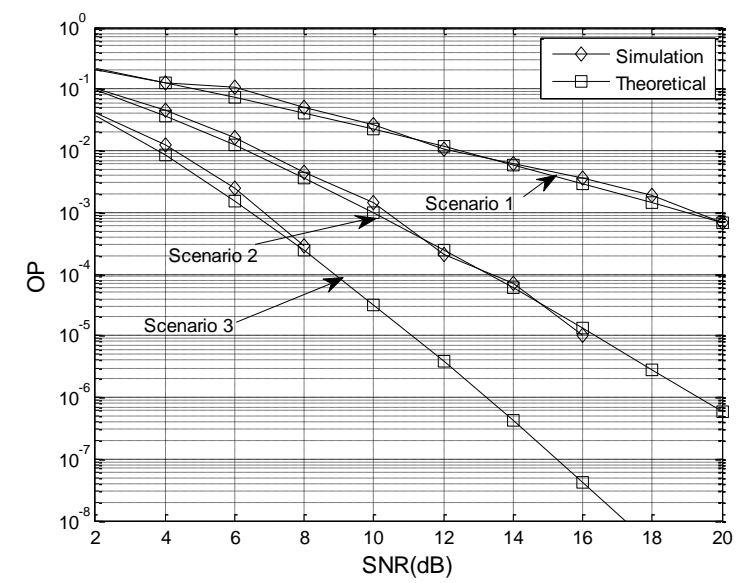

Figure 1. The OP Performance over N-Nakagami Fading Channels

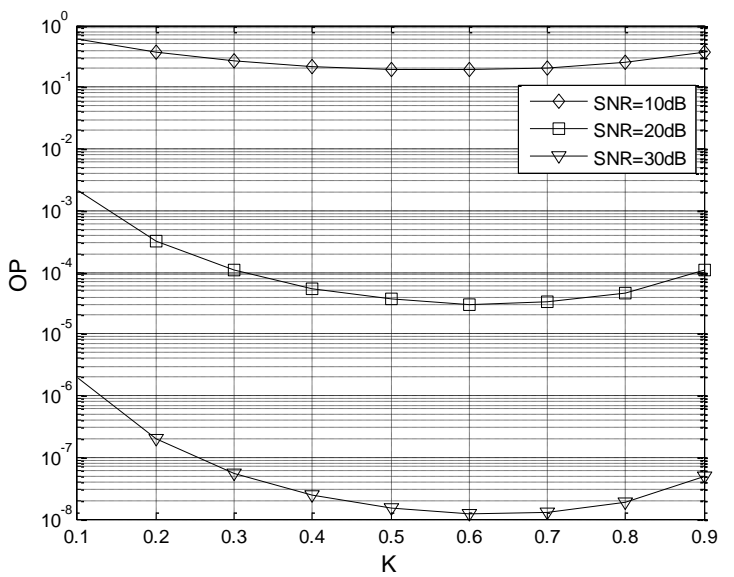

\section{Figure 2. The Effect of the Power-allocation Parameter $K$ on the OP} Performance

Figure 2 presents the effect of the power-allocation parameter $K$ on the OP performance of the home M2M networks over N-Nakagami fading channels with various values of SNR. The number of cascaded components $N=2$. The fading coefficient $m=2$. The relative geometrical gain $\mu=0 \mathrm{~dB}$. The given threshold $\gamma_{\mathrm{th}}=4 \mathrm{~dB}$. Simulation results show that the OP performance is improved with the SNR increased. For example, when $K=0.5, \mathrm{SNR}=10 \mathrm{~dB}$, the $\mathrm{OP}$ is $2 \times 10^{-1}, \mathrm{SNR}=20 \mathrm{~dB}$, the $\mathrm{OP}$ is $4 \times 10^{-5}, \mathrm{SNR}=30 \mathrm{~dB}$, the OP is $2 \times 10^{-8}$. When $\mathrm{SNR}=10 \mathrm{~dB}$, the optimum value of $K$ is 0.6 approximately; $\mathrm{SNR}=20 \mathrm{~dB}$, the optimum value of $K$ is 0.6 approximately; $\mathrm{SNR}=30 \mathrm{~dB}$, the optimum value of $K$ is 0.6 approximately. 


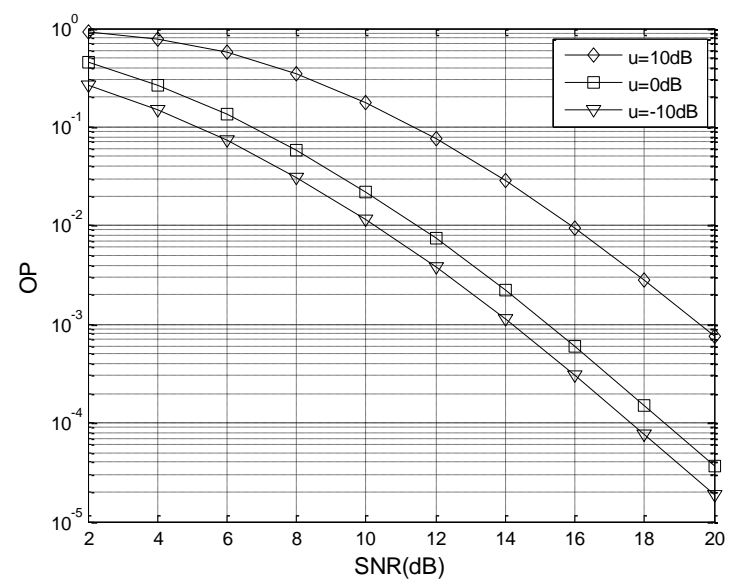

Figure 3. The Effect of the Relative Geometrical Gain $\mu$ on the OP Performance

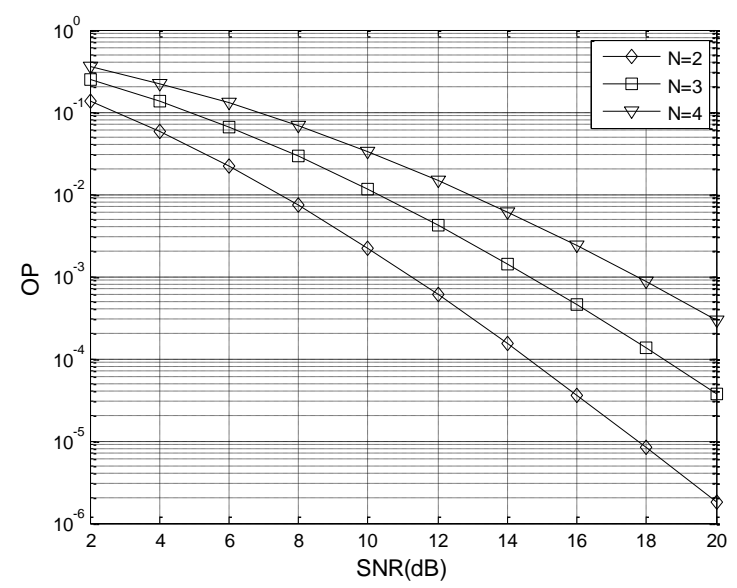

Figure 4. The Effect of the Number of Cascaded Components $N$ on the OP
Performance

Figure 3 presents the effect of the relative geometrical gain $\mu$ on the OP performance of the home M2M networks over N-Nakagami fading channels. The number of cascaded components $N=2$. The fading coefficient $m=2$. The given threshold $\gamma_{\mathrm{th}}=4 \mathrm{~dB}$. The relative geometrical gain $\mu=10 \mathrm{~dB}, 0 \mathrm{~dB},-10 \mathrm{~dB}$. The power-allocation parameter $K=0.5$.Simulation results show that the OP performance is improved as $\mu$ reduced. For example, when $\mathrm{SNR}=10 \mathrm{~dB}, \mu=10 \mathrm{~dB}$, the OP is $2 \times 10^{-1}, \mu=0 \mathrm{~dB}$, the OP is $2 \times 10^{-2}, \mu=-10 \mathrm{~dB}$, the OP is $1 \times 10^{-2}$. When the $\mu$ is fixed, with the increase of SNR, the OP is reduced gradually.

Figure 4 presents the effect of the number of cascaded components $N$ on the OP performance of the home M2M networks over N-Nakagami fading channels. The number of cascaded components $N=2,3,4$, which respectively denotes the 2-Nakagami, 3Nakagami, 4-Nakagami fading channels. The fading coefficient $m=2$. The relative geometrical gain $\mu=0 \mathrm{~dB}$. The power-allocation parameter $K=0.5$. The given threshold $\gamma_{\text {th }}=0 \mathrm{~dB}$. Simulation results show that the OP performance is degraded as $N$ increased. For example, when $\mathrm{SNR}=10 \mathrm{~dB}, N=2$, the $\mathrm{OP}$ is $2 \times 10^{-3}, N=3$, the OP is $1 \times 10^{-2}, N=4$, the OP is $3 \times 10^{-2}$.This because the fading severity of the cascaded channels increases as $N$ increased. When the $N$ is fixed, with the increase of SNR, the OP is reduced gradually. 


\section{Conclusions}

The exact closed-form OP expression for the home M2M networks over N-Nakagami fading channels is investigated in this paper. The simulation results show that: the fading coefficient $m$, and the number of cascaded components $N$, the relative geometrical gain $\mu$, and the power-allocation parameter $K$ have an important influence on the OP performance. The expressions derived here are simple to compute and thus complete and accurate performance results can easily be obtained with negligible computational effort. In the future, we will consider the impact of the correlated channels on the OP performance of the home M2M networks.

\section{ACKNOWLEDGEMENTS}

The authors would like to thank the referees and editors for providing very helpful comments and suggestions. This project was supported by National Natural Science Foundation of China (No. 61304222, No. 60902005), Natural Science Foundation of Shandong Province (No.ZR2012FQ021), Shandong Province Higher Educational Science and Technology Program (No.J12LN88), International Science \& Technology Cooperation Program of Qingdao (No.12-1-4-137-hz).

\section{Appendix A}

In the following subsections, we firstly evaluate $I_{1}$.According to (14), we can obtain

$$
P_{\text {outsr }}=\operatorname{Pr}\left(\frac{1}{2} \log _{2}\left(1+\gamma_{S R}\right)<R\right)=\operatorname{Pr}\left(\gamma_{S R}<\gamma_{t h}\right)
$$

where

$$
\gamma_{t h}=2^{2 R}-1
$$

So it can be given as

$$
\begin{aligned}
& \operatorname{Pr}\left(\gamma_{S R}<\gamma_{t h}\right)=\int_{0}^{\gamma_{t h}} f_{\gamma_{S R}}(x) d x \\
& =\frac{1}{\prod_{j=1}^{N} \Gamma\left(m_{j}\right)} \int_{0}^{\gamma_{t h}} \frac{1}{x} G_{0, N}^{N, 0}\left[\left.\frac{x}{\overline{\gamma_{S R}}} \prod_{j=1}^{N} \frac{m_{j}}{\Omega_{j}}\right|_{m_{1}, \ldots, m_{N}}\right] d x \\
& \overline{\gamma_{S R}}=K G_{S R} \bar{\gamma}
\end{aligned}
$$

To evaluate the integral in (21), the following integral function can be employed[12]

(19) can be given as

$$
\int_{0}^{y} x^{a-1} G_{p, q}^{m, n}\left[w x \mid \begin{array}{l}
a_{1}, \ldots ., a_{p} \\
b_{1}, \ldots, b_{q}
\end{array}\right] d x=y^{a} G_{p+1, q+1}^{m, n+1}\left[w y \mid \begin{array}{l}
\mid a_{1}, \ldots, a_{n}, 1-a, a_{n}, \ldots, a_{p} \\
b_{1}, \ldots, b_{m},-a, b_{m+1}, \ldots, b_{q}
\end{array}\right]
$$

$$
P_{\text {outsr }}=\frac{1}{\prod_{j=1}^{N} \Gamma\left(m_{j}\right)} G_{1, N+1}^{N, 1}\left[\left.\frac{\gamma_{t h}}{\overline{\gamma_{S R}}} \prod_{j=1}^{N} \frac{m_{j}}{\Omega_{j}}\right|_{m_{1}, \ldots, m_{N}, 0} ^{1}\right]
$$

We follow a procedure similar to (19) to yield as

$$
\begin{aligned}
& P_{\text {outsd }}=\operatorname{Pr}\left(\frac{1}{2} \log _{2}\left(1+\gamma_{S D}\right)<R\right)=\operatorname{Pr}\left(\gamma_{S D}<\gamma_{t h}\right) \\
& =\frac{1}{\prod_{j j=1}^{N} \Gamma\left(m_{j j}\right)} G_{1, N+1}^{N, 1}\left[\left.\frac{\gamma_{t h}}{\overline{\gamma_{S D}}} \prod_{j j=1}^{N} \frac{m_{j j}}{\Omega_{j j}}\right|_{m_{1}, \ldots, m_{N}, 0} ^{1}\right]
\end{aligned}
$$


where

$I_{1}$ can be given as

$$
\overline{\gamma_{S D}}=K \bar{\gamma}
$$

$$
I_{1}=\frac{1}{\prod_{j=1}^{N} \Gamma\left(m_{j}\right) \prod_{j j=1}^{N} \Gamma\left(m_{j j}\right)} G_{1, N+1}^{N, 1}\left[\left.\frac{\gamma_{t h}}{\overline{\gamma_{S R}}} \prod_{j=1}^{N} \frac{m_{j}}{\Omega_{j}}\right|_{m_{1}, \ldots, m_{N}, 0} ^{1}\right] G_{1, N+1}^{N, 1}\left[\left.\frac{\gamma_{t h}}{\overline{\gamma_{S D}}} \prod_{j j=1}^{N} \frac{m_{j j}}{\Omega_{j j}}\right|_{m_{1}, \ldots, m_{N}, 0} ^{1}\right]
$$

Next, we will evaluate $I_{2}$.

$$
\begin{aligned}
& \operatorname{Pr}\left(I_{S R} \geq R\right)=1-\operatorname{Pr}\left(I_{S R}<R\right) \\
&= 1-\frac{1}{\prod_{j=1}^{N} \Gamma\left(m_{j}\right)} G_{1, N+1}^{N, 1}\left[\left.\frac{\gamma_{t h}}{\gamma_{S R}} \prod_{j=1}^{N} \frac{m_{j}}{\Omega_{j}}\right|_{m_{1}, \ldots, m_{N}, 0} ^{1}\right] \\
& \operatorname{Pr}\left(\frac{1}{2} \log _{2}\left(1+\max \left(\gamma_{S D}, \gamma_{R D}\right)\right)<R\right) \\
&=\operatorname{Pr}\left(\gamma_{S D}<\gamma_{t h}\right) \operatorname{Pr}\left(\gamma_{R D}<\gamma_{t h}\right) \\
&=\frac{1}{\prod_{j j=1}^{N} \Gamma\left(m_{j j}\right)} G_{1, N+1}^{N, 1}\left[\left.\frac{\gamma_{t h}}{\gamma_{S D}} \prod_{j j=1}^{N} \frac{m_{j j}}{\Omega_{j j}}\right|_{m_{1}, \ldots ., m_{N}, 0} ^{1}\right] \times \\
& \frac{1}{\prod_{j j j=1}^{N} \Gamma\left(m_{j j j}\right)} G_{1, N+1}^{N, 1}\left[\left.\frac{\gamma_{t h}}{\overline{\gamma_{R D}}} \prod_{j j j=1}^{N} \frac{m_{j j j}}{\Omega_{j j j}}\right|_{m_{1}, \ldots ., m_{N}, 0} ^{1}\right]
\end{aligned}
$$

where

$I_{2}$ can be given as

$$
\overline{\gamma_{R D}}=G_{R D}(1-K) \bar{\gamma}
$$

$$
\begin{aligned}
& I_{2}=\left(1-\frac{1}{\prod_{j=1}^{N} \Gamma\left(m_{j}\right)} G_{1, N+1}^{N, 1}\left[\left.\frac{\gamma_{t h}}{\overline{\gamma_{S R}}} \prod_{j=1}^{N} \frac{m_{j}}{\Omega_{j}}\right|_{m_{1}, \ldots, m_{N}, 0} ^{1}\right]\right) \\
& \times \frac{1}{\prod_{j j=1}^{N} \Gamma\left(m_{j j}\right) \prod_{j j j=1}^{N} \Gamma\left(m_{j j j}\right)} G_{1, N+1}^{N, 1}\left[\left.\frac{\gamma_{t h}}{\gamma_{S D}} \prod_{j j=1}^{N} \frac{m_{j j}}{\Omega_{j j}}\right|_{m_{1}, \ldots, m_{N}, 0} ^{1}\right] \\
& G_{1, N+1}^{N, 1}\left[\left.\frac{\gamma_{t h}}{\overline{\gamma_{R D}}} \prod_{j j j=1}^{N} \frac{m_{j j j}}{\Omega_{j j j}}\right|_{m_{1}, \ldots, m_{N}, 0} ^{1}\right]
\end{aligned}
$$

\section{References}

[1] G. Wu, S. Talwar, K. Johnsson, N. Himayat and K. D. Johnson, "M2M: From mobile to embedded internet”, IEEE Communications Magazine, vol. 49, no. 4, (2011).

[2] E. Ko and S. Kim, "A Web Concurrency Control Agent running on a Home M2M Network", International Journal of Smart Home, vol. 8, no. 2, (2014).

[3] H. Munn, K. Lee, R. D. Caytiles and B. Park, "Network based Mobility Management for Smart Homes through Proxy Mobile IP", International Journal of Smart Home, vol. 8, no. 6, (2014).

[4] M. Uysal, "Diversity Analysis of Space-time Coding in Cascaded Rayleigh Fading Channels", IEEE Communication Letter, vol. 10, no. 3, (2006).

[5] F. K. Gong, P. Ye, Y. Wang and N. Zhang, "Cooperative mobile-to-mobile communications over double Nakagami-m fading channels", IET Communications, vol. 6, no. 18, (2012).

[6] G. K. Karagiannidis, N. C. Sagias and P. T. Mathiopoulos, "N*Nakagami: a novel stochastic model for 
cascaded fading channels", IEEE Transactions on Communication, vol. 55, no. 8, (2007).

[7] Q. H. Yang, Y. J. Zhong, K. S. Kwak and F. L. Fu, "Outage performance of opportunistic amplify-andforward relaying under Nakagami-m fading channels", ETRI Journal, vol. 30, no. 4, (2008).

[8] T. Q. Duong, V. N .Q. Bao and H. J. Zepernick, "On the performance of selection decode-and-forward relay networks over Nakagami-m fading channels", IEEE Communications Letters, vol. 13, no. 3, (2009).

[9] H. Ilhan, M. Uysal and I. Altunbas, "Cooperative Diversity for Intervehicular Communication: Performance Analysis and Optimization”, IEEE Transactions on Vehicular Technology, vol. 58, no. 7, (2009).

[10] F. K. Gong, J. Ge and N. Zhang, "SER Analysis of the Mobile-Relay-Based M2M Communication over Double Nakagami-m Fading Channels", IEEE Communications Letters, vol. 15, no. 1, (2011).

[11] W. F. Su, A. K. Sadek and K. J. R. Liu, "Cooperative Communication Protocols in Wireless Networks: Performance Analysis and Optimum Power Allocation", Wireless Personal Communications, vol. 44, no. 2, (2008).

[12] A. Papoulis, "Probability, Random Variables, and Stochastic Processes", McGraw-Hill, New York (1991).

[13] A. M. Mathai and R. K. Saxena, "Generalized Hypergeometric Functions with Applications in Statistics and Physical Sciences", Springer, New York, (1973).

\section{Authors}

Lingwei Xu was born in Shandong, China, in 1987. He received his Bachelor Degree in Department of Communication and Electronics, Qingdao Technological University, China, in 2011.From 2011 to now, he is a student of College of Information Science and Engineering, Ocean University of China. His research interests include ultra-wideband radio systems, MIMO wireless systems, and $60 \mathrm{GHz}$ wireless communications.

Hao Zhang was born in Jiangsu, China, in 1975. He received his Bachelor Degree in Telecom Engineering and Industrial Management from Shanghai Jiaotong University, China in 1994, his MBA from New York Institute of Technology, USA in 2001, and his Ph.D. in Electrical and Computer Engineering from the University of Victoria, Canada in 2004. His research interests include ultra-wideband radio systems, and MIMO wireless systems. He is a Professor in College of Information Science and Engineering, Ocean University of China.

Tingting Lu was born in Qingdao, Shandong Province, China, in 1983. She received her B.S degree in Communication engineering in 2006 from HuNan University, and she received her M.S. degree in Communication and Information systems and Ph.D in Computer application technology from Ocean University of China in 2009 and 2013 respectively. Now she is a lecturer at Ocean University of China. Her research interests are $60 \mathrm{GHz}$ wireless communication, and MIMO wireless communication.

T. Aaron Gulliver received the Ph.D. degree in Electrical and Computer Engineering from the University of Victoria, Victoria, BC, Canada in 1989. He joined the University of Victoria in 1999 and is a Professor in the Department of Electrical and Computer Engineering. In 2002 he became a Fellow of the Engineering Institute of Canada, and in 2012 a Fellow of the Canadian Academy of Engineering. His research interests include and communication theory, and ultra wideband communication. 
International Journal of Smart Home

Vol. 9, No. 4 (2015) 